\title{
Heart Failure Education of African American and Hispanic/Latino Patients: Data Collection and Analysis
}

\author{
Itika Gupta, ${ }^{1}$ Barbara Di Eugenio, ${ }^{1}$ Devika Salunke, ${ }^{2}$ Andrew D. Boyd, ${ }^{2}$ \\ Paula Allen-Meares, ${ }^{3}$ Carolyn A. Dickens, ${ }^{3}$ Olga Garcia-Bedoya ${ }^{3}$ \\ ${ }^{1}$ Department of Computer Science \\ ${ }^{2}$ Department of Biomedical and Health Information Sciences \\ ${ }^{3}$ Department of Medicine \\ University of Illinois at Chicago, Chicago, Illinois \\ \{igupta5, bdieugen, dsalun2, boyda\}@uic.edu \\ \{pameares, cdickens, ogarciab\}@uic.edu
}

\begin{abstract}
Heart failure is a global epidemic with debilitating effects. People with heart failure need to actively participate in home self-care regimens to maintain good health. However, these regimens are not as effective as they could be and are influenced by a variety of factors. Patients from minority communities like African American (AA) and Hispanic/Latino (H/L), often have poor outcomes compared to the average Caucasian population. In this paper, we lay the groundwork to develop an interactive dialogue agent that can assist AA and $\mathrm{H} / \mathrm{L}$ patients in a culturally sensitive and linguistically accurate manner with their heart health care needs. This will be achieved by extracting relevant educational concepts from the interactions between health educators and patients. Thus far we have recorded and transcribed 20 such interactions. In this paper, we describe our data collection process, thematic and initiative analysis of the interactions, and outline our future steps.
\end{abstract}

\section{Introduction}

Heart failure (HF) is defined as "a complex clinical syndrome that can result from any structural or functional cardiac disorder that impairs the ability of the ventricle to fill or eject blood" (Hunt et al., 2009). Approximately 5 million Americans currently live with this condition. In the United States, minority communities have a higher mortality rate than Caucasians (Roger, 2013; Toukhsati et al., 2019). This has been attributed to multiple factors like genetic variations, access to quality healthcare, socioeconomic conditions, health behavior, lower health literacy among others. However, some of these risk factors can be mitigated (Der Ananian et al., 2018; Tucker et al., 2011). For example, a patient with access to personalized educational material is better equipped to identify and address his self-care needs resulting in increased compliance and better health outcomes (Alberti and Nannini, 2013).

Self-care is "a naturalistic decision-making process by which individuals make choices about behaviors that maintain physiologic stability and the response to symptoms when they occur." (Riegel et al., 2004) However, this process can be rendered ineffective when the patient has a limited understanding of the disease. Furthermore, most selfcare materials available outside the hospital are catered towards the White Caucasian educated population, and thus lack cultural nuances to assist patients from minority communities (Barrett et al., 2019; Hughes and Granger, 2014; Lee et al., 2011). This has resulted in poor heart self-care regimen in minority communities (Howie-Esquivel, 2014).

Therefore, we intend to develop a dialogue agent that can provide medically reliable and culturally sensitive self-care information to discharged African American and Hispanic/Latino HF patients, and help mitigate the health disparities observed among them. In this paper, we talk about our first step towards building the agent i.e. collecting the data (since there is no publicly available dataset) and analyzing it. We used topic modeling to identify core educational concepts and analyzed the data for initiative, i.e., who takes the conversational lead. Not surprisingly, educators take more initiative, however the portions in which the patient has control are more important to uncover what patients may ask of a dialogue agent.

We also tried to evaluate the interactions for cultural competency. However, existing tools such as Cross-cultural counseling inventory (LaFromboise et al., 1991) and the Multicultural counseling inventory (Sodowsky et al., 1994) focus on provider's knowledge and do not evaluate patient educational materials. Therefore, with the help of content experts in our team, fundamental concepts of crosscultural care (empathy, respect, and curiosity), and 
focus groups (Bobo et al., 1991; González-Lee and Simon, 1987), we will manually identify culturally relevant topics and model the dialogue agent accordingly as part of our future work.

\section{Related Work}

In the 1960s, ELIZA was the first Natural Language Processing (NLP) based chatbot which facilitated a dialogue between humans and machines. Since then, multiple advances have been made in artificial intelligence and NLP resulting in the evolution of dialogue agents. They have transitioned from accepting very restricted user input (answers to multiple-choice questions only) to processing full sentences and providing medically reliable information (Laranjo et al., 2018).

Multiple randomized control trials have established the efficacy of dialogue agents in healthcare settings as well (Bickmore et al., 2013a,b; Lovell et al., 2017). They have been successfully used to promote a healthy lifestyle, increase adherence, or provide adjunct psychotherapy among other uses (Laranjo et al., 2018). Technology-based interventions have been used to assist HF failure patients for quite some time. Most of these interventions are catered towards remote monitoring and medication management (Hughes and Granger, 2014). CARDIAC (Computer Assistant for Robust Dialogue Interaction and Care), a conversation assistant for chronic HF patients was designed to collect both objective and subjective information from the patients (Ferguson et al., 2009). Similarly, DIL, another conversation agent was designed to help HF patients to transition from hospital to their homes by motivating them to follow a healthy lifestyle and maintaining medication adherence (Moulik, 2019). To our knowledge, there is no existing culturally sensitive dialogue agent designed to assist minority communities with their heart failure self-care needs.

\section{Data Collection}

We recruited three health educators to conduct HF education of $\mathrm{AA}$ and $\mathrm{H} / \mathrm{L}$ patients in both the inpatient and outpatient clinics of our university. We plan to collect $40 \mathrm{HF}$ education sessions, half with AA patients and half with $\mathrm{H} / \mathrm{L}$ patients. We have recorded 20 interactions so far, 18 with AA patients and two with $\mathrm{H} / \mathrm{L}$ patients; of these 20 patients, 8 are males and 12 females. One of the barriers to recruiting $\mathrm{H} / \mathrm{L}$ patients is how our hospital iden-
Patient: Yeah, I don't, I don't do the frozen meal.

Educator: Okay.

Patient: I was basically doing the uh, vegetables.

Educator: Okay.

Patient: Frozen vegetables,

Educator: They should be fine.

Patient: Yeah.

Educator: But... but, but I do want you to start looking at those nutrition labels.

Patient: Okay.

Educator: And look for something that says less than 5\%.

Patient: Okay.

Educator: So, the other thing we always want you to do is, um, of course take all your medicines like you're supposed to. Patient: Which I didn't do last night.

Educator: Okay.

Figure 1: Excerpt from a conversation

\begin{tabular}{|c|c|c|c|}
\hline \multicolumn{4}{|c|}{20 transcripts } \\
\hline & turns & sentences & words \\
\hline Educator & 116.90 & 205.45 & 2281.10 \\
Patient & 108.40 & 131.20 & 849.50 \\
Total & 225.30 & 336.65 & 3130.60 \\
\hline
\end{tabular}

Table 1: Distributional analysis of the conversations

tifies them; additionally, since at the moment we focus on English as the language of interaction, we exclude $\mathrm{H} / \mathrm{L}$ patients if the interaction is conducted in Spanish. Lastly, H/L patients comprise only $20 \%$ of our hospital population; this is less than half of the AA patients (45\%). The remaining 35\% comprises 10\% Asian American, and 25\% Caucasian and others.

All the 20 recordings were transcribed by a professional transcription service. An excerpt is shown in Figure 1. We should note that in some cases, a third person (a caregiver, like a spouse) is present, and the conversation may involve both patient and caregiver, or be mostly between the educator and the caregiver. While transcribers did a good job, they failed to capture linguistic practices and choices of patients (vernacular speech) and converted it to standard English: for example, 'gonna' was transcribed as 'going to'. Given our focus is on cultural sensitivity, such linguistic practices are of great importance to us, and therefore, the transcripts were revised again to make sure that exactly what was said is recorded.

The average length of an interaction is about 15 minutes. Table 1 presents the average number of turns, sentences, and words per conversation over these $20 \mathrm{HF}$ education sessions. A turn refers to a complete unit of speech and can consist of multiple sentences. Therefore, it makes sense that 

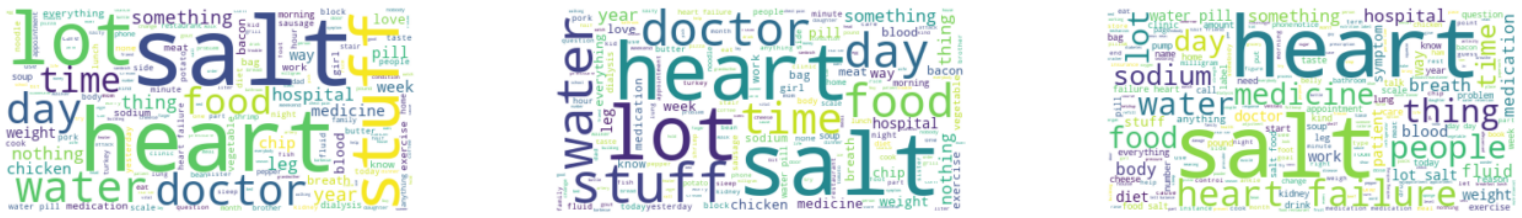

Figure 2: Word cloud of common nouns: both educators and patients, the patients, and the educators (left to right)

\begin{tabular}{|ll|l|}
\hline & Interpretation & $\begin{array}{l}\text { Example words from } \\
\text { top 10 }\end{array}$ \\
\hline \hline 1 & low salt diet & food, sodium, label, meat \\
\hline 2 & symptoms & pill, fluid, weight, swell \\
\hline 3 & $\begin{array}{l}\text { explaining heart fail- } \\
\text { ure }\end{array}$ & $\begin{array}{l}\text { pump, failure, blood, } \\
\text { body }\end{array}$ \\
\hline 4 & $\begin{array}{l}\text { medication and follow- } \\
\text { up appointment }\end{array}$ & $\begin{array}{l}\text { appointment, medication, } \\
\text { hospital, doctor }\end{array}$ \\
\hline
\end{tabular}

Table 2: Interpretation of LDA topics

\begin{tabular}{|l|l|}
\hline patients & educators \\
\hline eating, cooking, restaurant, & eating, health, cooking, liq- \\
health, liquid, ocean, sailing, & uid, sailing, ocean, water, \\
water, children, speaking, & speaking, restaurant, com- \\
negative_emotion, medi- & munication, healing, giving, \\
cal_emergency, shopping, & medical_emergency, busi- \\
party, communication & ness, cleaning \\
(same count as healing) & \\
\hline
\end{tabular}

Table 3: Most common categories in order of frequency

both the educator and patient had a similar number of turns except the cases where the caregiver did some of the talking. However, there is a noticeable difference in the number of sentences and words between the two. The educator spoke 2.5 times more words than the patient. This indicates that the educator did most of the talking during the interviews. Figure 2 shows the most common nouns in the conversations as word clouds. The frequency of words provides some indication of the topics of conversation, but we now turn to a deeper analysis of these interactions.

\section{Data Analysis}

In this section, we will look at two types of analysis: thematic analysis and initiative analysis.

Thematic analysis looks at the most common topics of discussion related to HF education. The educators aimed to provide comprehensive information about HF to the patients so that they can take care of themselves upon discharge. The educators covered topics such as what HF is, the role of medications such as the "water pill" (a diuretic), the importance of a low sodium diet, the benefits of physical activity, daily checks to recognize symptoms, and the value of follow-up appointments. The topics were motivated by the standard HF education information that should be given to a HF patient. However, it is the discussion that arises from these topics that varies based on culture and even person to person. Therefore, we used existing topic modeling tools to further analyze the transcripts.

We first performed topic modeling using Latent Dirichlet Allocation (LDA) (Blei et al., 2003). We used the Gensim library consisting of python wrapper for LDA from MALLET, the java topic modeling toolkit. We tried topic modeling for both the educator and the patient turns together and separately. Since the educators did most of the talking, and almost $50 \%$ of patient turns involved filler sentences such as 'okay', 'umm', the patient turns in themselves weren't sufficient to learn separate topics. The remaining $50 \%$ of patient turns focused mostly on salt/food which was one of the recurring topic words during the experiments. Therefore, we decided to model the educator turns exclusively. After experimenting with a different number of topics and alpha parameters (Dirichlet prior on the per-document topic distributions), we found the coherence score was the highest for topics counts = 4 and alpha $=0.01$. We show the output in Table 2 .

We then used Empath to identify the difference in topics between the patients and the educators (Fast et al., 2016). Empath is a text analysis tool that can help identify various topical and emotional categories present in a text. It consists of 200 builtin categories and allows to create more categories on demand. We analyzed both educator and patient turns separately using the built-in categories and show the topmost common 15 categories in Table 3. We used the raw counts for categories in empath. The categories in bold indicate categories different between the educators and the patients. One can notice that 11 of the categories are the same and the most common is eating. This is consistent with the word clouds where salt and food were two of the most common nouns.

However, it is interesting to notice that patients 
Control: Patient _................. C

Patient: Yeah, I don't, I don't do the frozen meal. (assertion/command)

Educator: Okay. (prompt)

Patient: I was basically doing the uh, vegetables. (assertion/command)

Educator: Okay. (prompt)

Patient: Frozen vegetables, (assertion/command)

-.......... Control: Educator _..........

Educator: They should be fine. (assertion/command)

Patient: Yeah. (prompt)

Educator: But... but, but I do want you to start looking at those nutrition labels. (assertion/command)

Patient: Okay. (prompt)

Educator: And look for something that says less than 5\%. (assertion/command)

Patient: Okay. (prompt)

Educator: So, the other thing we always want you to do is, um, of course take all your medicines like you're supposed to. (assertion/command)

-......... Control: Patient ...........

Patient: Which I didn't do last night. (assertion/command) Educator: Okay. (prompt)

Figure 3: Example conversation showing utterance type and control transfer.

prioritized children in their discussion (family was also in the top 20 categories), whereas for educators neither of them were even in the top 25 categories. negative_emotion, shopping, and party were in the top 20 of educator categories, therefore can be considered similar to patient categories where they are in top 15. Lastly, giving, business, and cleaning categories were more common in educators. This is because giving relates to the term 'give' which educators used frequently to provide information such as 'give you a followup appointment', 'give you a phone number to call', 'give you medicine'. Business relates to terms such as 'need', 'work', and 'company', which, similarly to giving, was used to inform patients about different companies offering low sodium salt, what they need to do upon discharge, and to educate them about how medications work. Cleaning is in the top categories because it relates to the term 'water' which can be considered a partial duplicate of category water.

Initiative analysis focuses on the distribution of turns based on the person taking the lead in the conversation. A person takes the lead/initiative when he/she contributes to the conversation (e.g., by asking a question) instead of only answering the questions or responding with fillers (such as 'okay', 'umm'). In turn, when a speaker takes initiative, the control of the conversation transfers to that speaker and remains with the same speaker until the other speaker takes initiative.
We classified a given turn as a question, prompt, or assertion/command where: a question tries to elicit information, a prompt doesn't express any propositional content, an assertion states facts, and a command intends to instigate action (Walker and Whittaker, 1990). We used the rules below to automatically annotate the turns:

- Question: if the turn ends with a question mark (?)

- Prompt: if a turn consists only of words 'uhhuh', 'okay', 'ok', 'yeah', 'umhmm', 'right', 'oh', 'umm', 'uh', 'hmm', 'umumm', 'ummm', 'alright'

- Assertion/Command: everything else

We didn't separately annotate command and assertion as we were more interested in the number of questions and prompts by the educators and patients; additionally, it would be hard to distinguish them using simple rules.

The rules for control transfer used by us are shown below (Turn type: Controller):

- Assertion/Command or Question: speaker unless response to a question

- prompt: hearer

Figure 3 shows the excerpt from Figure 1 marked with utterance type and control transfer. The utterances with type assertion/command indicate speaker initiative. On analyzing the transcripts, we found that on an average per conversation, educators asked 26 questions and produced 17 utterances with prompts as compared to 3 questions and 39 prompt utterances by the patients. As a consequence, an educator held the initiative for about 95 turns per conversation, whereas the patient did for 51 turns; the control lasted for about 5 turns on average in the case of an educator as compared to patients who only held control for 2 turns on average. These observations about patient/educator interactions have also been confirmed by an expert we have consulted with, Dr. Kishonna Gray from Department of Communication and Gender and Women's Studies at University of Illinois Chicago.

We hypothesize that, even if few, the turns where the patient takes control are important for the development of the dialogue agent: in fact, we envision this dialogue agent as an assistant that the patient will have to interact with on their initiative, rather 
than a system that operates as a health educator per se. Next, we will extract the topics from the turns where the patients have control since those are probable topics of discussion. We also plan to conduct focus groups with 10 self-identified AA and $10 \mathrm{H} / \mathrm{L}$ patients to gain insight into their lives post HF diagnosis and evaluate the acceptability of a dialogue agent to discuss HF. We believe talking to individuals with HF outside the hospital environment can help solicit questions that do not appear in the recordings or existing literature.

\section{Conclusions and Future Work}

In this paper, we discussed our data collection process for heart failure education conversations between educators and African American or Hispanic/Latino patients. We analyzed 20 transcribed recordings and found that the most common topic of discussion was food. Patients also discussed family and children frequently. Though mostly educators took the lead, we will extract topics where patients take control to build a dialogue agent that can answer patient queries effectively. We will also use insights from these interactions to inform the questions for the focus groups which we will conduct in the future.

\section{Acknowledgments}

We would like to thank Dr. Kishonna Gray for her valuable feedback and ideas on how to capture the cultural and inter-sectional complexities present with Hispanic/Latino and African American patients. This work is supported by the University of Illinois Chicago Discovery Partners Institute (DPI) Seed Funding Program.

\section{References}

Traci L. Alberti and Angela Nannini. 2013. Patient comprehension of discharge instructions from the emergency department: A literature review: Patient comprehension of discharge instructions from the ED. Journal of the American Academy of Nurse Practitioners, 25(4):186-194.

Matthew Barrett, Josiane Boyne, Julia Brandts, HansPeter Brunner-La Rocca, Lieven De Maesschalck, Kurt De Wit, Lana Dixon, Casper Eurlings, Donna Fitzsimons, Olga Golubnitschaja, Arjan Hageman, Frank Heemskerk, André Hintzen, Thomas M. Helms, Loreena Hill, Thom Hoedemakers, Nikolaus Marx, Kenneth McDonald, Marc Mertens, Dirk Müller-Wieland, Alexander Palant, Jens Piesk, Andrew Pomazanskyi, Jan Ramaekers, Peter Ruff,
Katharina Schütt, Yash Shekhawat, Chantal F. Ski, David R. Thompson, Andrew Tsirkin, Kay van der Mierden, Chris Watson, and Bettina Zippel-Schultz. 2019. Artificial intelligence supported patient selfcare in chronic heart failure: a paradigm shift from reactive to predictive, preventive and personalised care. EPMA Journal, 10(4):445-464.

Timothy W. Bickmore, Daniel Schulman, and Candace Sidner. 2013a. Automated interventions for multiple health behaviors using conversational agents. $\mathrm{Pa}$ tient Education and Counseling, 92(2):142-148.

Timothy W. Bickmore, Rebecca A. Silliman, Kerrie Nelson, Debbie M. Cheng, Michael Winter, Lori Henault, and Michael K. Paasche-Orlow. 2013b. A randomized controlled trial of an automated exercise coach for older adults. Journal of the American Geriatrics Society, 61(10):1676-1683.

David M Blei, Andrew Y Ng, and Michael I Jordan. 2003. Latent dirichlet allocation. Journal of Machine Learning Research, pages 993-1022.

Loretta Bobo, Robin J Womeodu, and Alfred L Knox Jr. 1991. Society of general internal medicine symposium principles of intercultural medicine in an internal medicine program. The American Journal of the Medical Sciences, 302(4):244-248.

Cheryl Der Ananian, Donna Winham, Sharon Thompson, and Megan Tisue. 2018. Perceptions of heart-healthy behaviors among african american adults: a mixed methods study. International Journal of Environmental Research and Public Health, 15(11):2433.

Ethan Fast, Binbin Chen, and Michael S Bernstein. 2016. Empath: Understanding topic signals in largescale text. In Proceedings of the 2016 CHI Conference on Human Factors in Computing Systems, pages 4647-4657.

George Ferguson, James Allen, Lucian Galescu, Jill Quinn, and Mary Swift. 2009. CARDIAC: An Intelligent Conversational Assistant for Chronic Heart Failure Patient Heath Monitoring. In 2009 AAAI Fall Symposium Series.

Teresa González-Lee and Harold J Simon. 1987. Teaching spanish and cross-cultural sensitivity to medical students. Western Journal of Medicine, 146(4):502.

Howie-Esquivel. 2014. A culturally appropriate educational intervention can improve self-care in hispanic patients with heart failure: a pilot randomized controlled trial. Cardiology Research.

Hannah Anderson Hughes and Bradi B. Granger. 2014. Racial disparities and the use of technology for selfmanagement in blacks with heart failure: a literature review. Current Heart Failure Reports, 11(3):281289. 
Sharon Ann Hunt, William T. Abraham, Marshall H. Chin, Arthur M. Feldman, Gary S. Francis, Theodore G. Ganiats, Mariell Jessup, Marvin A. Konstam, Donna M. Mancini, Keith Michl, John A. Oates, Peter S. Rahko, Marc A. Silver, Lynne Warner Stevenson, and Clyde W. Yancy. 2009. 2009 focused update incorporated into the ACC/AHA 2005 guidelines for the diagnosis and management of heart failure in adults. Journal of the American College of Cardiology, 53(15).

Teresa D LaFromboise, Hardin LK Coleman, and Alexis Hernandez. 1991. Development and factor structure of the cross-cultural counseling inventory-revised. Professional psychology: Research and practice, 22(5):380.

Liliana Laranjo, Adam G Dunn, Huong Ly Tong, Ahmet Baki Kocaballi, Jessica Chen, Rabia Bashir, Didi Surian, Blanca Gallego, Farah Magrabi, Annie Y S Lau, and Enrico Coiera. 2018. Conversational agents in healthcare: a systematic review. Journal of the American Medical Informatics Association, 25(9):1248-1258.

Christopher S. Lee, Debra K. Moser, Terry A. Lennie, and Barbara Riegel. 2011. Event-free survival in adults with heart failure who engage in self-care management. Heart \& Lung, 40(1):12-20.

Karina Lovell, Peter Bower, Judith Gellatly, Sarah Byford, Penny Bee, Dean McMillan, Catherine Arundel, Simon Gilbody, Lina Gega, Gillian Hardy, Shirley Reynolds, Michael Barkham, Patricia Mottram, Nicola Lidbetter, Rebecca Pedley, Jo Molle, Emily Peckham, Jasmin Knopp-Hoffer, Owen Price, Janice Connell, Margaret Heslin, Christopher Foley, Faye Plummer, and Christopher Roberts. 2017. Low-intensity cognitive-behaviour therapy interventions for obsessive-compulsive disorder compared to waiting list for therapist-led cognitive-behaviour therapy: 3-arm randomised controlled trial of clinical effectiveness. PLOS Medicine, 14(6):e1002337.

Sanjoy Moulik. 2019. DIL - A Conversational Agent for Heart Failure Patients. Ph.D. thesis.

Barbara Riegel, Beverly Carlson, Debra K Moser, Marge Sebern, Frank D Hicks, and Virginia Roland. 2004. Psychometric testing of the self-care of heart failure index. Journal of Cardiac Failure, 10(4):350-360.

Véronique L. Roger. 2013. Epidemiology of heart failure. Circulation Research, 113(6):646-659.

Gargi Roysircar Sodowsky, Richard C Taffe, Terry B Gutkin, and Steven L Wise. 1994. Development of the multicultural counseling inventory: A self-report measure of multicultural competencies. Journal of Counseling Psychology, 41(2):137.

SR Toukhsati, Tiny Jaarsma, AS Babu, Andrea Driscoll, and DL Hare. 2019. Self-care interventions that reduce hospital readmissions in patients with heart failure; towards the identification of change agents. Clinical Medicine Insights: Cardiology, 13:117954681985685.

Carolyn M. Tucker, Michael Marsiske, Kenneth G. Rice, Jessica Jones Nielson, and Keith Herman. 2011. Patient-centered culturally sensitive health care: Model testing and refinement. Health Psychology, 30(3):342-350.

Marilyn Walker and Steve Whittaker. 1990. Mixed initiative in dialogue: An investigation into discourse segmentation. In Proceedings of the 28th annual meeting on Association for Computational Linguistics, pages 70-78. Association for Computational Linguistics. 